the solution a little stronger than the powder, putting generally in 100 parts, six, eight, or ten of arsenic.

Case.--In March, 1833, a young girl, 15 years of age, entered the hospital to be treated for a suspicious-looking ulcer on the lower lip. It was about an inch in circumference ; the edges deep and inverted, and the base considerably indurated. The disease had commenced seven years before, and continued daily to increase in magnitude; part of the ulcer was covered by a yellow crust, the rest presented a pretty deep cavity. It seemed to me to have arisen from a complication of the scrofulous and cancerous diatheses, with a venereal taint. The sore was first cleaned by poultices, and the arsenical powder applied, which caused a slight inflammation. In a few days, the paste being removed, we found the surface of the ulcer improved; a second application of the remedy scemed to give rise to some symptoms of poisoning, as the patient was affected with nausea and vomiting. These accidents were combated by a strict diet, and the exclusive use of milk as food; the symptoms soon disappeared, and on the removal of the crust formed by the powder, the ulcer was almost completely healed. Indeed you saw her a few days afterwards in the lecture-room, when nearly every trace of disease was removed.

In similar cases I take care to give abundance of milk to the patient, and place a piece of lint over the sore, giving particular orders that it be not removed until the powder has completely become dry. You have seen me use the same means in the case of a man who was affected with an eating ulcer of the lip and nose, with whom this mode of treatment was equally successful. The marner of applying the remedy is extremely simple. We first clean the surface of the ulcer by poultices, and then touch the sore with a small bit of lint, charged with the powder, so as to cover its surface with a layer of the arsenic, if the ulcer be not large; but when of great extent, it is prudent not to cover more than a third or half, according to circumstances. The solution, or more properly speaking the paste, is applied in the same way with a spatula. The application, after having cansed some considerable pain, inflammation, \&c., usually comes away at the end of eight or ten days: it should be renewed according to the exigency of the case, until the complete cure of the ulcerated surface is obtained. This commonly takes place in five or six applications, and often two or three are sufficient. This remedy, which I have used for fifteen years, under the two forms described to you, acts not as a cautery, but as an alterant of the diseased surface. I have never seen it produce eschars, or destruction of the part to which it was applied; it is infinitely less adherent than the pastes of Frere Come or of Rousselot, and causes much less pain and tumefaction.

\section{GANGLION OTICUM ARNOLDI.}

No. I.-Remarks on the Discovery of the Otic Ganglion;

No. II. - Description of the Otic GANGLION;

Read at the London Medical AssociaTION, Westminster General Dispensary, August 9th, 1833, (Dr. Epps, Chairman,)

$$
\text { By John Thurnam, Esq., London. }
$$

\section{No. I.}

IN introducing a paper to the consideration of this infant, but (as I trust it will prove) very useful association, it affords me much pleasure to endeavour to promote the advancement of its objects, and at the same time to gratify the great interest which I feel in the subject which I have selected. For were I asked what part of the human frame I considered more admirable in its structure, more beautiful in its arrangement, and, conse. quently, more attractive as a study than another, I should unhesitatingly reply,the nervous system.

Let us examine the brain. Let us liken it to the magnificent metropolis of some great and ancient $\in$ mpire, with its spacious streets, its palaces, and its temples, where the mind, as supreme, bears unquestioned and all-powerful sway, and from which city numerous noble roads diverge to every part of her dominions, along which roads are constantly passing a mighty array of agents, commissioned to execute alike her highest and her lowest behests; whilst, from the frontiers of her territories, other agents are returning with incredible velocity, bringing intelligence of every passing event which can in the least degree affect the interest of her throne.

This is a figurative, but a correct description of this wondrous part of the frame, and as such, I hope it will animate us to continue our travels along, and become well acquainted with, all its beaten tracks, and in proportion as we do so, shall we hail with delight the discoverer 
who penetrates into a newly-discovered ed to me necessary to premise this much forest, or explores a nameless sea. The respecting the sympathetic in general, in names of a Bell, of an Arnold, or of a order to our properly considering its rela. Tiedemann, will rejoice us. tions to the organs of the cxterual senses,

I sincerely trust, that anatomy, as the into which I now proceed to inquire.

very corner-stone of our science, will receive every encouragement and attention from this association.

In offering these remarks, it is with the intention rather of directing the course of the evening's debate, and of raising, instead of settling, the points of discussion. With this intention I shall first make a few gencral observations on the organic or sympathetic system of nerves in general; then inquire whether a ganglion of this system loes notbelong to the eye; thence argue the necessity for a corresponding one to the functions of the ear: and, lastly, in order to make the subject complete, shall briefly consider the relations of this part of the nervous system to the three remaining organs of senise-the nose, the tongue, and the cutis.

The system of nerves of organic or vegetable life, or of the great symparhetic, appears to be of primary importance. We find that its nerres are distributed principally to those organs the almost, if not constant, pertormance of whose functions are absolutely necessary to life. These functions are, respiration, assimilation, circulation, and secretion. Their great importance probahly led the Hunter of France-Bichat-to call the ganglia of this system "little brains," thus investing them, figuratively perhaps, with the power of perception. But though this is ingenious, it totally fails to grive us any idea of the manner in which these ganglia and their nerves act. And who shall solve the difficulty? Will the ingenious theory of the indefatigable Swan, not badly smportel either by pathology, experiment, or reasoning, satisfy us? Can we believe that the impulse of the circulating blood is the constant stimmlus to these nerves, and that it is for this purpose that they are distributed to the onter coats of the arteries; that the heart (heing the primum suliens) and the arterics give activity to those nerves, and that they are thus qualified to act constantly and uniformly on the organs which receive their final distribution? Whatever may be the defects of this theory, it has at least the merit of supposing a canse quite adequate to the effects produced, so far as constancy of action is necessary. However, I fear we shall scarcely adopt this view, ingenious as it may be as a theory, and that it must be acknowledged, that as to the source of the power possessed by these ganglia, we know nothing. It seem-

Without entering at all into the anatomy of the eye, I shall merely allude to those motions of the iris which occasion a dilatation or a contraction of the pupil, motions which we know to be quite independent of the will. We know that these motions are caused, not by any effect produced on the iris directly by light, but only through the medium of the retina. Now how did the elder anatomists account for any effect produced in this indirect manner? They told us it was by sympathy. But this ineant nothing, uniess there were a medium through which they could sympathise; ayd until Tiedemann published his valuable discovery, that the ciliary nerves ramify in the retina as well as the iris, we could not satisfactorily ac. count for it. Well, then, it appears that these ciliary nerves are the agents which, being either sub or superabundantly stimulated through the medium of the retina by the light, cause a corresponding involuntary motion of the iris, which produces either a dilatation or a contraction of the pupil. Mark that I say "inro. luntary;" for a great excess of light produces a very different and a voluntary motion of the eyelids. Whence do these ciliary nerves proceed, and does their anatomy prove then to be nerves of the system of organic life? We find them emerging from the anterior border of a very small ganglion in the orbit, the ophthalmic or lenticular. Posteriorly we find two filaments leaving it, the shorter of which is traced uniting to the inferior oblique branch of the third or motor oculi nerve, whilst the loncer passes backwards, griided by the nasal branch of the ophthaluic division of the fifth nerve; then on the ophthalmic itself, till, lastly, reaching the Gasserian ganglion, it jasses downwarls to the carotil plexns, which ascends from the first cervical ganglion. This sketch of the anatomy of this ganglion is sufficient to prove that it forms part of the system of the great sympathetic.

Having shown the necessity for these nerves in the eye, the question proposes itself in relation to the present subject, Does the mechanism of the ear require any corresponding set of involuntary nerves?

Like the immediate seat of sight-the retina, - the membranous or nerrous ear is seated deeply, having a rather complicated mechanism intervening between it and the air. It will suffice here to ob- 
serve, that the membrana tympani ap- lingual ganglia. Whether by the " nasal pears to be to the ear what the iris is to ganglion," he means the Spheno-pulative the eye-the sentinel which prevents an or Meckel's Ganglion, I can scarcely decide, undue access of sound to the interrial ear. though I should presume so. But as the But here a difficulty presents itself, for external mechanism, both in the nose and Bichat tells us, that it is relaxed by pow- the tongue, is extremely simple, and aperful, and rendered tense by weaker, pears to require adaptation (as in the eye sounds. Now we shall find by this paper, and ear) to external circumstances, we that Savart's experiments prove the re- can hardly see the necessity for such inverse. But to proceed, how are these voluntary nerves, and neither can we motions of the rympanum effected? Is reason physiologically on their function. the true membrana tympani muscular, as Sir E. Home supposed? We should incline to say no, when we perceive that there exist certain muscles whose functions appear to be to effect the motions of this membrane. The principal muscle of th these is the tensor tympani. We find, then, the greatest analogy between the mechanism of the ear and the eye. We have found a coritrivance which can effect the motions of the tympanum, had it nerves corresponding to the ciliary, without which we well know no muscular contraction can take place. We want a ganglion as a nervous centre, precisely corresponding with the ophthalmic, with nerves ramifying with the auditory in the membranous ear, and others supplying the muscles of the tympanum. And all this we shall find in the ganglion oticum.

Though the names of parts, expressive of structure and of function, are in every point of view to be preferren to those derived from the name of their discoverer or describer, yet as custom sind the authority of Tiedemann sanction the name of G. Amoldi, we shall gladly associate his name with those of Meckel, Cloquet, and Ribes, the illustrious discoverers of the other ganglia of the crganic system in the head. It would be a great service to anatomy, were the translator of the present part* encouraged to translate the whole work, as the relations of the rest of the cephalic portion of these nerres still require much elucidation and accurate description. Indeed, till very recently in this comtry, i.e., till the publication of the two now most deservedly popular works, the "Dublin Dissector," and Professor Quain's work, many of these ganglia were described as ganglia of the cerebro-spinal system, and their functions quite misunderstood.

It appears from the paper of Arnold, that he regards the other cerebral ganglia of this system as having a relation corresponding with the two other organs of sense in the head, - the nose and the tongue, - as he speaks of the nasal and

As, however, the analogy of structure remains, it is probable that the mechanism eluded us from its minuteness.

The submaxillary ganglion, situated behind the gland of that name, is probably the one he means when he speaks of lingual, and may have some such relation to the tongue. Before leaving the tongue I would observe, that that organ is largely supplied with nerves; but I would inquire which is the nerve of taste? Here is an interesting field for inquiry, for it has no nerve which is not possessed by other respiratory muscles. In the present state of our knowledge of the functions of the nerves, I presume we must consider the sense of taste to be that of touch, highly developed.

The last of the organs of the external senses is the cutis, the organ of touch. This sense being possessed in a greater or less degree by every part of the surface of the body, accounts for the great number of primary nerves communicating it. It is so important, that the external parts of all the other organs of sense are provided with it; so that it might with propriety be termed a universal sense, but it has, very improperly, been termed " common sensibility," and the nerves ministering to it, " nerves of common sensation." To quote the language of the eminent elucidator of the nerves, Sir Charles Bell, as used at the Royal College of Surgeons, "There is no such a thing as common nerves and common sensibility. Different nerves have different properties; different parts have distributed to them nerves which bestow, never common, but a wonderfully appropriate and peculiar degree of, sensibility."

If nerves of organic life be requisite to the mechanism of the cutis, in order to the due performance of its functions, we must seek for them in the coats of the arteries distributed thereto. We know that they exist in the outer coat of all the larger arteries, and the probability is that they accompany them to their extremest distribution; for these nerves appear to have a universal connexion with the rest of the nervous system. 
No. II.

A Description of the OTIC Ganglion, abridged from an Article in the "Edinburgh Medical and Surgical $J_{\text {out.," for }} J_{u l} l y, 1833$, entitled, "Anatomical and Physiological Description of the Otic Ganglion; extracted from Arnold's ' Kopftheil des Vegetativen Nervensystems bcien Menschen' (the Cephalic part of the Vegetative System in Man), and translated from the German, by W. S. Tylee, Esq., Devizes."

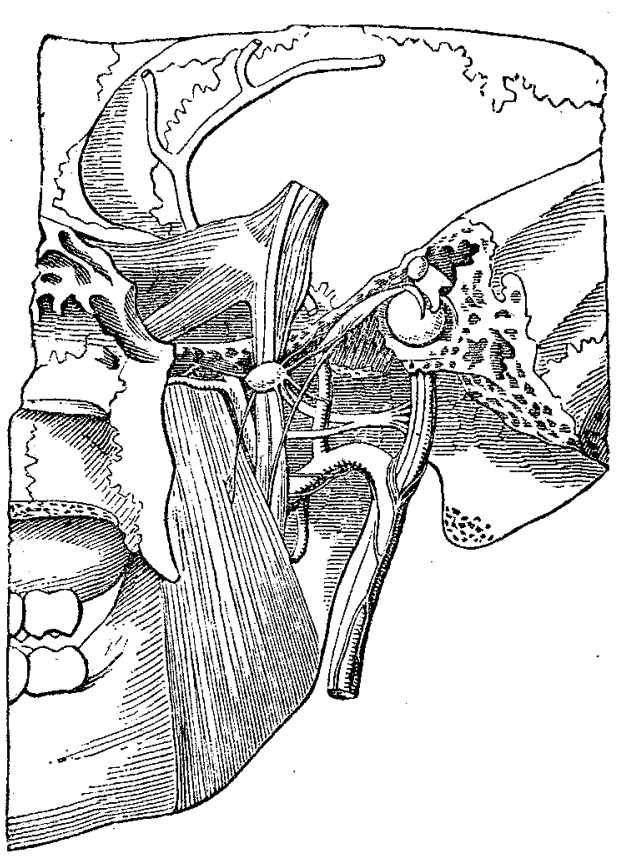

The Ganglion Oticum sive Auriculare, called also by Professor Tiedemam, Ganglion Arnoldi, is situated at the internal side of the third branch of the trigeminus, immediately under the foramen ovale, at the spot where, externally, the nervi temporales profonde, nervus maxillaris, and buccinatorius, branch off, and where the smaller portion or anterior root of this nerve unites more closely with the larger branch, or posterior root from the Gasserian ganglion.

Internally, the ganglion is covered by the cartilaginous part of the eustachian tube and the origin of the tensor palate. Behind, it borders on the arteria meningea media. It has an oval figure, flattened on either side, and a little elongated horizontally, in which direction it measures, on an average, from two to two and a half lines; from above downwards from one and a half to two lines; and in thickness only a quarter to, or, at most, half a line. It is characterized by its reddish colour, verging rather towards gray. It is surrounded by a very fine and delicate neurilema, which is pretty closely connected with the substance of the ganglion. In this envelope ramify its vessels, which, in proportion to its size, are very numerous, and to which it doubtless owes much of its reddish character. These all come from the arteria meningea media, or directly from the internal maxillary and enter its posterior, and partly its inferior, border.

The ganglion is imbedded, particularly externally, in a quantity of cellular and adipose tissue, and by which it is united to the internal surface of the third branch of the trigeminus.

On examining the structure of the ganglion it is found to consist of a reddishgray ganglionic substance. Numerous white nervous filaments are, however, interwoven with this, which are to be traced, some from the pterygoid nerve of the third branch of the trigeminus, which, on a superficial dissection, is in so close connexion with this ganglion as to appear to arise from it; but on more accurate in. vestigation it is clear that this nerve, soon after its origin, penetrates through and takes up a part of the substance of the ganglion. Others of these filaments are traceable from that branch of the nervus tympanicus of the glossopharyngeal, which I call nerv. petrosus superficialis minor. Many other filaments enter it from the small or muscular portion of the nerv. trigem., and (except where the ganglion is rather distantly sitriated, when of course they will be longer and more easily observed) they are mostly extremely short and only to be observed when we try to separate the ganglion from the nerve, with which, as we have seen, it is also in close cellular connexion.

Thus it is clear, that the ganglion oticum communicates with the portio minor quinti paris, also with the nerv. gloss. pharyng. by means of the nerv. tympanic, and indirectly by means of the same nervous branch with the facial and auditory nerves. This last connexion is important, and is effected by a filament, which, joining the nerv. petros. superf. min., is to be traced to the gangliform swelling of the facial nerve, situated at its curvature, from which swelling it is also found proceeding to the acoustic nerve.

We have now to describe those nerves which have their origin from this ganglion. They appear soft, reddish, and delicate, and agree in their qualities altogether with the ganglion. The most important, which must be principally attended to, as showing the particular relation of this 
ganglion to the organ of hearing, arises at its upper and posterior border, and takes its course along the internal side of the art.mening. med., backwards and upwards ; penetrates into that part of the eustachian tube in which the tensor tympani is situated, and ramifies in that muscle. This thread distinguishes itself essentially from the nerv. petros. superf. minor, near which it directs its course. There are other small nerves of the same character, which show still more its relation to the ear. They arise at its inferior and posterior border, and proceed to the bifid root of the anterior auricular nerve from the third branch of the trigeminus. These, probably, communicate with the branches of this rerve, supplying the external auricular canal and membrane of the tympanum.

We thus find, that the otic and ophthalmic ganglions agree very much in situation. form, size, and structure, inasmuch as the former is situated by a similar relation to the third branch of the trigeminus, as the latter is to the first branch. They also each communicate with two different nerves of the animal system, one for motion and another for sensation, by means of branches which they receive from them; for the short but considerable filaments from the small portion of the trigeminus, subservient to the motions of mastication, correspond with the short root of the ophthalmic ganglions, coming from the nervus oculo-motorius, and the long root of the same, which is given off by, a nerve of sensation, we find corresponding in the otic ganglion with the nerv. petros. superf. min. coming from the gangliform swelling of the gloss. pharyng. nerv., which is likewise, at least in some degree, a nerve of sensation.

\section{On the Functions of the Otic Ganglion.}

It is not merely the anatomical character of the otic ganglion in the human subject, and its analogy to the ophthalmic ganglion, so far as it has a relation to the organ of hearing, and especially to the membrane of the tympanum, similar to that which the other has to the eye, and particularly to the iris, that justify us in concluding the otic ganglion to have the same office in the organ of hearing that the ophthalmic ganglion has in the eye.

Researches in comparative anatomy render it also most probable, that the ganglion in question is the central organ for the involuntary motions of the membrane of the tympanum. The result of these researches is,-First, That the otic ganglion is only to be found in those animals which have a tensor tympani. As this muscle does not, according to my re- searches, belong to birds and the amphibials, but is to be found in the mammalia only, so these alone possess the ganglion in question. Secondly, These researches teach us with certainty that this ganglion is larger, and more intimately and multifarionsly connected with the organ of hearing, the larger and more developed the external ear is. Indeed, in those animals in which the ear is very large and moveable, it is double. This I found to be the case in the hare, rabbit, horse, and pig, all of which,"and especially the former, have extremely long ears. Those mammalia which have a tolerably developed external ear-as, for instance, the ruminating animals, possess a rather large but single otic ganglion; whilst in those whose external ear is but moderately developed, as the cat, dog, marten, hamster, and rat, the otic ganglion is much smaller. Lastly, we find this ganglion smallest and most inconsiderable where there is no external ear, as in the mole, in which it is not found without the greatest care and patience.

From these two points, namely, that the otic ganglion only exists in those animals which have a tensor tympani, and that it is so much the larger, more developed, and more intimately connected with the organ of hearing,-the larger and more developed the external ear is, it clearly results that this ganglion must be considered as the central organ for the involuntary motions of the membrane of the tympanum; for it only exists where there is an apparatus for the tension of this membrane, and is more intimately connected with the ear,--the more numerous are the sounds received by a large and moveable external ear. From this we see evidently, that the tension of the membrane of the tympanum (by which, as we shall presently show, the effect of a too powerful sound on the ramification of the auditory nerve is moderated) is, and must be, so much the more considerable, the greater the quantity of sound received from all directions by the external ear, so varied in its formation.

The difference with respect to the motions of the membrane of the tympanum must first be considered. Those which take place in consequence of an irritation reflected from the acoustic nerve on the apparatus for moving the membrane of the tympanum, are to be distinguished from those which take place from the fluctuation of the air. The latter are the real motions according with this fluctuation; but the former consist more in a simple tension and relaxation of this membrane, which is produced by a too powerful action of sound on the ramifications of the 
auditory nerve being imparted by means|nerves. So, also, from the influence of of the communication of this nerve with powerful sounds on the nervous thread the facial, and the connexion of the latter ramifying with the acoustic nerve, this with the smaller superficial petrous nerve affection is conmunicated to the otic ganto the otic ganglion, which, according to glion, from which a reaction takes place the impressions received, exercises such on the tymparum, and changes equal to an influence on the tensor tympani, that the degree of sound ensue. But it seems the membrane is put into a degree of ten- to me very probable, that when sounds sion according to the power of the sound, in order to prevent a too violent irritation of the auditory nerve.

It appears to me that sufficient attention has not hitherto been paid to distinguish accurately and decidedly the difference in the motions of the membrane of the tympanum, in so far as they appear in one way as vibrations, and in another as a tension and relaxation of that membrane. Indeed the vibrations of the tympanum produced by sound, have been called automatical motions. But here a signification has been given to the word automatical which does not at all belong to it; for such of its motions as depend only on external influences, and ensue immediately from them, cannot be called automatical; but we can only designate with this name those which are produced under the influence of the otic ganglion by the apparatus for moving the above membrane, and must, doubtless, distinguish them from those motions which consist in vibrations of the membrane, and are produced immediately by the fluctuation of the air.

My anatomical investigations having proved the communication of the acoustic nerve with the otic ganglion, enable us not merely to see in what way sounds, acting powerfully on the acoustic nerve, are imparted to the apparatus for moving the membrane of the tympanum, but also lead us no longer to consider the brain as the organ through which that communication takes place, in those cases at least where the influence of sound is not very powerful. According to Tiedemann's valuable discovery, ${ }^{*}$ branches from the ophthalmic ganglion communicate with the optic nerve and retina; in like manner, also, a thread communicating with the otic ganglion joins the acoustic nerve, and ramifies, doubtless, with it, in the labyrinth of the ear. As in cases where the irritation from light is not too considerable, by the immediate affection of the ciliary nerves, which spread themselves in the retina, and without the mediation of the brain, motions are produced in the iris corresponding with the different degrees of light, in consequence of a reaction of the ciliary ganglion on these

- Zeitschrift fur Physio'ogie, Bd. 1, H. 2. act too powerfully, so that the ear is unpleasantly affected, the reaction may follow through the brain, as it is not merely that we are conscious of an unpleasani sensation, hut parts which are under the control of the will come into action; for in such cases we close our eyelids and draw up the under jaw, and thus lessen the area (lumen) of the external auditory passage.

We now have to inquire the reasons of the several communications of the otic ganglion with the several nerves.

I believe the reason of its indirect coms munication with the acoustic nerve through the medium of the facial to be, that the latter supplies with nerves the muscles of the external ear, and that thus their mo* tions are made to correspond with those of the tympanum. These muscles are, indeed, of slight importance in the human subject, having but little influence on the motions of the ear; but as they are large and important in many mammalia, we must not, for this reason, overlook the importance of this union.

The intimate connexion of the otic ganglion with the third branch of the trigeminus, enables one of its branches, the superficial temporal, supplying the external auditory canal and membrane of the tympanum, to communicate impressions made on those parts, to the otic ganglion, especially as this branch, arising from the posterior root of this branch of the trigeminus, must be regarded as a nerve of sensation.

Its communication with the masseter, temporal, and pterygoid branches from the anterior or motor root of this same nerve (port. min. quint. par.), enables the ganglion to communicate the necessary inpressions to those nerves, in order to their effecting a contraction correspond. ing with that which it produces directly in the tensor tympani, in the muscles of the lower jaw, thus pressing its condyloid process against the inferior parietal portion of the external ear, contracting it a little, and thus impeding the sound.

Its communication with the glossopharyngeal nerve appears to be owing, in the first place, to that nerve giving off a filament supplying the glandulus ceruminosus auris, and anastonosing with the posterior auricular of the seventh pair; 
and, secondly, to the same nerve, supplying so freely, in the form of the nervus tympanicus, the cavity of the tympanum.

We thus perceive, from what has been said, that the otic ganglion, through various communications, has the most intimate relation to the organ of hearing, and that its office, with regard to the tensor tympani, is of the greatest importance. These points, the results of researches in comparative anatomy, and also the ana$\log y$ to the ophthalmic ganglion, clearly show that this ganglion must be considered as the central organ for the automatical motions of the nembrane of the tympanum.

\section{CAST OF}

\section{TIC DOULOUREUX}

CURED BY THE

\section{"MINERAL MAGNET."}

By E. S. Budndeli, M.D., London.

A LADY, ${ }^{*}$ ætat. 53 , of a robust constitution, enjoyed a tolerable share of health until a few years since, when she was seized one morning, on awakening, with a violent pain in the left side of the lower jaw, directiy undor the bicuspid tocth, which continued without intermission for several days. She applied to her medical attendant, who cousidered it to be a rhenmatic aficction, the result of an exposure to cold during an evening ride. The remedies employed were, hot fomentations, followed by stimulating anodyne liniments, for the space of ten days, without any sensible mitigation of the patient's sufferings. At the end of this period a physician was consulted the fomentations were omitted, and leeches were applied, and he prescribed a saline mixture, with Dover's ipowder. These remedies were persisted in for a fortnight, with no better result than attended the exhibition of the former.

The pain increasing in intensity and duration, spread from the jaw to the ear, and from thence to the biscuspid of the upper jaw, and again across the face to the ear, and from thence to the temple. The agony of the patient at this period was great; she was neither able to masticate, swallow, nor speak, without aug-

* The name and address of this lady may be obtained, on application to Dr. Blundell, by any respectable jerson who may wish a reference to the patsent herself.

No. 521 . menting her sufferings. The disease heing now pronounced a decided case of tic-douloureux, the treatment was changed. Carbonate of iron was administered for the space of twenty days, in doses commencing with one ounce, and gradually increasing to six ounces per diem, without producing any favourable results. During the last four or five years, morphia, quinine, belladona, strychnine, turpentine, lobelia, apcrients, counter-irritation, and arsenic, were each and severally applied without the slightest benefit.

In July, the patient called on me, and placed herself under my care, in order that 1 mighit try the effect of the magnetic trcatmont. Her pulse was 85 ; tongue pale and puffy; skin hot and dry ; countenance anxious, with an icterial tinge; bowels irritable, with tenesmus after each evacuation; the eyes were glassy, and her general appearance was haggard. She stated that she had not had more than half an hour's sleep at a time for the last six weeks. The pain at each attack was confined to the inner orbit of the left eye, upper and lower lip, temple, and behind the ear.

July the 10th. This morning I commenced the application of the magnet, by passing it over the parts affected, for ten minutes. The patient was sensibly relieved. In an hour the spasms returned with increased intensity for a few minutes. After again passing the magnet, she was perfectly easy for four hours. At 10 o'clock I visited her, and found her free from pain. The application of the instrument was repeated every twelve hours from the 11th to the 17th with the most decided benefit. During this period, a generons and rather stimulating diet was enjoined, and under this treatment the patient's general health and appearance rapidly improved. From the 18 th to the 23 rd the magnet was only applied once a day, and on the 24th I repeated the application for the last time. The patient returned home on the 25 th, and up to the present date has not experienced any return of the malady.

In this interesting case I could reproduce the pain in the merve, and transfer it from the head to the extremity of the littlefinger of the affected side, and vice versa thus giving a striking illustration of the motion (I had almost said the circulation) of the nervous or vital principle along those mysterious conductors.

\section{3, Orchard-st., Portman-square,} 20 th August, 1833. 